\title{
DESIGN SPACE MACHINERY
}

\author{
ROBERT F. WOODBURY, ANDREW L. BURROW \\ Simon Fraser University, Vancouver, CANADA \\ RMIT University, Melbourne, AUSTRALIA \\ rw@sfu.ca, andrew.burrow@ems.rmit.edu.au
}

\begin{abstract}
Design space exploration is a long-standing focus in computational design research. Its three main threads are accounts of designer action, development of strategies for amplification of designer action in exploration and discovery of computational structures to support exploration. Chief amongst such structures is the design space - the network structure of related designs that are visited in an exploration process. There is relatively little research on design spaces to date. This paper sketches a partial account of the structure of both design spaces and research to develop them.
\end{abstract}

\section{Design Space Exploration}

Design space exploration is the idea that computers can be used to help designers by representing many designs, arraying them in a network structure (the space part) and by assisting designers to make new designs and to move amongst previously discovered designs in the network (the exploration part). This conceptual complex arose from some of the initial ideas that occurred to researchers in design computing. Over the years, the complex has been built through descriptive research on designing, prototype systems, thought experiments, mathematics, and considerable scholarly debate. Today it rests on three premises. Each of these is itself an area of research.

The first premise is that exploration is a good model for designer action. Numerous studies have shown the utility of modelling designers as information processing systems that search in order to satisfy goals in a strongly constrained problem space. Furthermore, humans are endowed with specific and limited cognitive structures that constrain their behaviour as searchers and representers of problem spaces. A feature of this research is that it is explained in terms of production; that is, its aim is to reproduce and extend the behaviour of designers.
The second premise is that designers would find useful tools that amplify their abilities to represent goals and problems spaces, and to search for designs. Researchers have created a modest number of computer programs that attend to such amplification tasks. One of these, KIRTS/GENESIS [1] has seen industrial application. The results provide weak evidence for the utility of such amplification.

The third premise is that computational support for exploration is feasible. Namely, that there are tractable representations and algorithms that provide suitable amplification for design explorers. A large body of literature provides support for this premise. There exist numerous formalisms for representing designs, for acting on designs to produce other designs, and for recording such action. A large secondary literature uses such mechanisms to produce relatively compact generative descriptions of corpora of existing and conjectured design work.

Research in design space exploration takes on several distinct patterns. Typically, it addresses representation, search algorithms, task description, or interaction design. Relatively little work focuses on the design space itself. Instead, most research focuses on design states and on making action explicit. Little work exists that depends upon computational access to the design space $[2,3,4,5]$. Yet, it would appear that the design space itself is where the largest gains are to be made. Designers typically consider a very small number of alternatives in their work, and this is explained by cognitive limits.

Why the paucity of research on the design space? Conjecture is easy, and the debate is open. Clearly, prior work on representation and action devices is a prerequisite for serious thought about design spaces. Furthermore, any useful computational account of a design space requires both mathematical sophistication and deep knowledge of the design domain. There may be easier pickings elsewhere or it may be that design space exploration has limited utility. There is probably truth in all of these conjectures. 
In recent years a group of researchers, initially at Adelaide University, but now distributed across Australia, Canada and Taiwan, has developed a representation for design spaces that is computable (and thus formal) and appears to have sufficient informal expressiveness to capture complex aspects of designs. The representation builds on a formalism from computational linguistics, Carpenter's [6] typed feature structures, and extends it to be an account of the design space. To researchers in conventional design grammars, the formalism appears strange as it substitutes types for rules, a unification-based resolution procedure for generating designs, and a set of composable navigation operators for complex moves in design space. It does posit an account of the indefinite parts that are declared essential in the shape grammar literature [7]. It is a highly regular system as a consequence of aiming at disciplined generation, navigation and reuse. It has certain striking limits that are a consequence of its attainment of efficient algorithms for key computations.

The present paper explores some of the implications of having such a design space representation. It does this in reference to each of the three premise areas: designer action, the utility of amplification and the symbolic representation and computation of design spaces. Its main focus is on designer action; it presents brief sketches of the arguments for amplification and computation.

\section{Designer Action}

The metaphor of designers as finite explorers entails both designer abilities and the necessary structure of exploration. In this section, we take a brief look at each, focusing on the two key issues of representation and designer situation in an incomprehensibly large space of possibilities. We find that good representations for design are invariably both partial and intentional; and that accessibility is a key to addressing the huge size of design space.

There are traps in building an account of designer action suitable as a basis for a design space explorer. One is the highly programmatic thinking that bedeviled early attempts to build expert systems for design. By programmatic, we mean the overly literal attention to representing and computing directly over the concrete entities in the problem at hand. To be fair to the field, almost all serious expert systems for design were built on two-levels: a lower abstract inference engine and an upper representation of problem particulars. For example, R1 [8] was built on top of OPS [9]. LOOS [10] originally in OPS, and GENESIS [11] on a hybrid logic/solid-modelling substrate. The trap opened when the specific domain representation was taken too seriously. To put criticism close to home, the early thinking of researchers in the SEED project [12] confused the knowledge level (involving such things as functional units, design units and technologies) with underlying computations that could support it. The result of falling into this trap is to miss two crucial points: good representations are, in a deep sense, both intentional and partial, and good accounts of designing are given in terms of the most general constructs possible.

If we are committed to realising designs as physical artefacts, then ultimately we are committed to their intentionality. That is, we are committed to the notion that designs are about other objects. This level of indirection, the aboutness of designs, becomes a problem when we conflate the properties of a design with the properties of its subject. Representation theory tells us that these must be different. This conflation has facets.

First, a designed object will embody phenomena beyond the reach of any particular representation. Thus, we do not find airflow around buildings accounted for in a typical building model, nor do we find explicit reference to the classical motifs used ironically by Charles Moore in representations of his work, and especially seldom do we find represented the ineffable sense of space and light that is the hallmark of much fine architecture. Therefore, what we can actually infer and what we can "do" with a representation is necessarily limited by those aspects of its referent that it captures. Yet, as designers, we must constantly make decisions in just such explicitly unrepresented realms.

A second facet is that we use representations in distinct ways, which we will label here, strong and weak representation. Strong representation occurs when we imbue in the representation the ability to make algorithmic inference about its referent. A ubiquitous example is the now near-universal use of 3D modelling in architectural schools. The chief inference here is, of course, visualisation. Students (and designers in practice) have found that the effort involved in building a 3D model is more than offset by the ability to multiply render the resulting design. Another example, more prosaic, but perhaps even more ubiquitous, is the spreadsheet. With it, the quick quantitative model of a budget, an architectural brief or a plan of work, has become has become an essential part of much professional practice. Cognitive accounts of designing tend to focus on the strong aspects of a representation, as these are precisely the ones amenable to the constructive nature of the theory-building at hand with its requirement for computationally executable theories. 
Weak representation happens when we use a representation as a reminder, hint or framer of new insight. Situationist accounts of designing focus mostly on such weak aspects of representation, largely and precisely because such have been mostly ignored by the cognitivists.

A third facet is that, to use representations computationally, we must imbue them with exogenous properties, that is, ones that are manifestly not in the phenomena they purport to represent. We have to do this to use representations - the fact that designers work by search in a problem space means that they have to be able to alter representations and most such alterations do not follow the logic of physical construction processes. Good design representations are inherently about supporting change precisely to help designers move from idea to idea in their search.

A good representation to some degree must avoid all of these traps. It must not overcommit to a specific and limited set of represented properties and feasible inferences. It should display some aspects of strong representation, else a significant part of the benefit of using a computer is lost. It must remain open to the weak uses of representation, or designers will resolutely suborn it to such. Lastly, it must afford a disciplined notion of change, else it is not a design representation and certainly not a computable one.

Partiality swims with intentionality. A design is about an artefact. That is no commitment to being all about the artefact, and especially no commitment to being as much about the artefact as it might some day become. Designs are inherently partial — we add to them and subtract from them as a routine part of doing design. Much of the long debate about the overly determinate aspects of CAD representation swirls around just this issue. Furthermore, aboutness harbours information that may itself be partial - representations may refer to the same or distinct objects. For instance, we might have two identical representations of a column in a building, each playing a role in some part of a structural system, yet these two representations might be identified as referring to a single structure in a future version of the representation. Such function-sharing is one of the oftcited sources of design innovation.

The domain tells us more. Design spaces are Vast in the sense meant by Dennett [13]. While almost all conceivable classes of designs are also Vast, they remain a Vanishingly small part of the entire design space, so that randomly selecting a worthwhile design is an event with a Vanishingly small probability. Yet, without an oracle, we must begin at just such a random place and proceed to search. In the analogous context of Darwin's theory of evolution, Dennett [13] argues that expressiveness does not give an adequate account of possibility - the grade of possibility that matters is not whether a suitable artefact is expressible as a design, but whether a suitable design is accessible with reasonable effort to an explorer positioned somewhere in the design space. This line of reasoning can be expected to apply to other Vast design spaces. Therefore, the explored fraction of a design space matters precisely because it decides the cost of accessing the unexplored designs.

Figure 1 demonstrates two dimensions of accessibility. Distance in the figure is a measure of derivational effort. Arcs link designs in the explored part of the space to designs not yet found. Following an arc expands the explored part of the space. First, effort counts. Design $b$ will be more accessible than design $a$. Second, connectivity counts. Designs $c$ and $d$ gain an advantage over designs $a$ and $b$ through their connection to a larger number of already discovered designs.

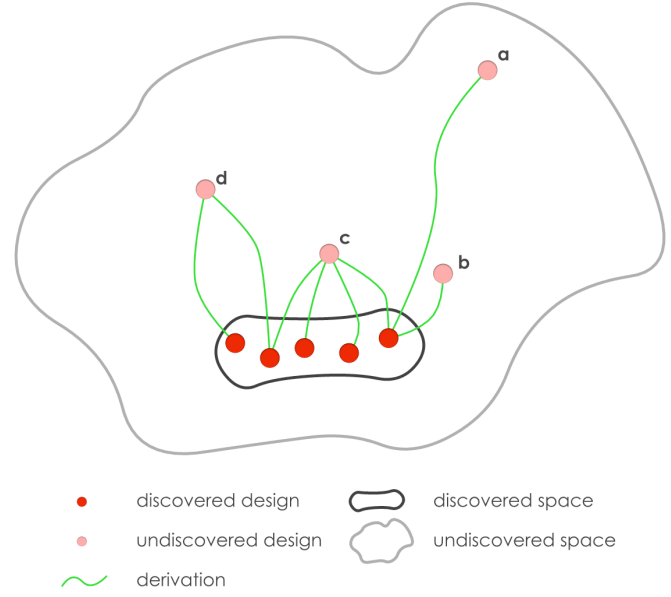

Figure 1: Dimensions of design space accessibility

Vastness has other implications to the implied mechanism of search. Effort counts in other ways than making designs accessible along short paths. Design is time-pressured - the fact of Vastness of the space and finite human life guarantees this. The effort required to formulate a proposal is large relative to the time available for the entire process. Designs are precious productions and designers do return to them, to use them directly or to infer from them analogous structures and moves that can be applied to other designs. In terms of a design space, designers pull tendrils of actuality from existing designs and apply them to others in the space. In the time pressured world of designing. Even partial and inconsistent designs find value as launching points into unexplored parts of the space. Designs themselves are important objects, irrespective of what they represent. That they are placed in the design space captures a position from which further exploration can be made. This is a corollary of the fact that accessibility 
is the measure of possibility-designs without physical interpretation or with poor qualities may be the basis for other realisable designs. While this matter is also related to the expressiveness of the formalism, particularly whether it allows the description of abstract forms, the role of unsound designs derives from the importance of accessibility in the design space. Designs without satisfactory interpretations draw their utility from the designs that they make accessible either by forming a link in a chain of explored designs, or by providing a chain of exploration that may be reused by analogy.

The primary design action of search in a space deeply colours the accounts of design intent that will be feasible. We can equally dismiss a posteriori rationalisations of design intent as self-serving and atomistic efforts to include intent with overly strong representation. Intent is contextual and the context is the thread of design space along which it comes into play. Design spaces enlarge the context for design decisions beyond the brief initiating the exploration. Just as design space accessibility stands for possibility, the correct context for interpreting the intent of design decisions is the position in the design space. When asking the why question concerning the telos of a feature of a design, or of the resulting artefact, the most satisfactory explanation will include the design space trajectory and the alternatives forsaken. The brief changes in the process of exploration-designs create the context for further decisions on them and the design space trajectory records alternative paths considered. Thus a move in the design space can be interpreted as seeking a new solution to a particular problem arising in some previous design, where in general the representation of the problem and its solution may remain implicit in both the antecedent and consequent designs. Put another way, the co-option of earlier features for new roles is a part of design that can only be understood with respect to the design space trajectory. In analogy to Gould's [14] claims about the necessity of history in any specific evolutionary account design intention finds its most powerful explanations through a contingent history of process. As a specific example, justifications for residential air-conditioning outside of the tropics come not solely from a desire for human comfort but also from the design decisions proposing a building configuration producing uncomfortable thermal environments.

We see from both professional and scholarly literatures that derivation is a productive form of explanation. It is a recurrent theme in the generative design literature [15]. It is a typical device in papers reporting grammars for design corpora. After the grammar rules are introduced, comes the application of the rules to produce one or more designs in the space.
This part of the exposition helps the reader knit together an understanding of the rules in joint action.

Designer action changes with experience. This is most commonly called learning. The process has explicit aspects: in mature learners at least, learning itself becomes an object of cognate action Schön [16]. Under the standard cognitive claims about the production-like quality of cognition, it would hardly be surprising if such a production-like quality surfaced in first person accounts of design learning. It does [17, 18, 19]. In summary, designers use production-like encodings of extant and newly-learned actions. They use such encodings both internally and in communication with colleagues.

We claim that the above features of design representations are invariants of the exploration view and are necessary properties of a useful design space representation. In summary representations must avoid the programmatic pitfall, must be intentional and partial, are inevitably limited in scope, carry both strong and weak representational qualia, and have properties exogenous to the artifact represented. That representations are used in search in a Vast space makes accessibility the primary criterion of utility, drafts unsound designs into a potentially crucial role and points to contingent historical accounts of design history as the best record of design intent.

Simon long argued that the limits of cognitive capability would seldom surface in observing problem solving action [20]. Here surely is a case where the absence of evidence should not be taken as evidence of absence. Entirely absent from unaided human problem solving are strategies that require the storage of even modest numbers of problem states. Indeed the absence of such strategies forms a profound falsification test for cognitive theories of problem solving. Discovery of a single instance of such a strategy would deeply challenge the entire edifice. Ubiquitous is the choice of designers to use external memory of some form as an aid to work. It turns out that external memory, of almost any form, is a significant amplifier of action. What is not, and cannot be, clear from accounts of designer action alone is the utility of providing computational means for designers to surmount these biological limits. Can designer action be so amplified?

\section{Action Amplification}

Simon notes repeatedly that we should expect to see primarily the properties of the task environment when we observe designer action. Except in extreme situations we do not see the situations in which cognitive limits are reached as problem solvers simply avoid such situations. 
Yet is precisely at such places where human cognition limits action that we should seek to amplify human ability. In addition, we know they exist. Since, with a few exceptions [21, p 826] we do not have positive empirical accounts of designer limitations, we need to resort to argument and anecdote to build a catalog of opportunity. Presuming that humans find amplification of their abilities useful, we should expect to find opportunity at the boundaries of systems and we sketch two extant examples of these below. The first is conventional and is found in the rationale and utility of almost every commercial CAD system. It appears here more as an existence proof for amplification than as the focus of design space explorers. The second, codification, is the strategy behind both scripting languages for CAD systems and the early rule-based generative design systems. We leave our hypotheses for new amplifiers to a later article.

\subsection{REPRESENTATIONAL PROWESS}

External memory use is near-ubiquitous for designers and representation of designs is a primary function for external memory. A good account of the history of commercial design computing can be built on events introducing new representational tricks. One such trick was solid modelling, the representation of the geometry of three-dimensional solid objects. At its inception, solid modelling was seen as a key support technology for a larger enterprise of supporting design with computers. ${ }^{1}$ This happened, but as things played out, boundary representation solid modelling also gained a life of its own and is now, in and of itself, a key design medium. Why did this occur? It turned out that solid modelling was Just Enough to give designers a playground and not Too Much to overburden them with specifics. The operations typically provided (abstractly, primitive parametric solids, boundary tweaking and the Boolean operators) allowed designers to make virtually any form they could conceive. There is no meaning captured beyond solidity; designers are free to make their own associations between the forms represented and the thing being designed. Rendering allowed designers to play with visual effect far beyond simple realistic veridicality with the world. Solid modelling with computers is the analog to the model-making shop still to be found in many design schools.

\footnotetext{
${ }^{1}$ At a first approximation, we can make this claim without citation. One of the authors (Woodbury) was a participant in this process.
}

\subsection{CODIFICATION}

Numerous authors (both designers reflecting on their work and scholars observing designers) note processes of codification of design moves. These vary in temporal extent from within a single design episode [22] through individual projects $[15,16]$ to constancies and changes through the development of a life's corpora [23]. Designers preferentially use past successful moves (their own or others) in future projects. One of the long promises of grammars is the ability to explicitly encode such moves.

Such encoding coheres with the ability of computers to be active with data. Many (most?) professional tools come with a programming language that can be used to extend the tool to automate tasks. Apparently, explicit codification of action is important. Very few such languages support the production-like character that we noted above as a feature of much designer communication on design process. A partial exception is the venerable AutoLISP, recently reborn as Visual Lisp, which at least has structures that make production like expression relatively easy. Designers and firms do use these codification tools, though seldom to the degree that academic purists might prefer.

The main strategy for amplification in early grammar formalisms and interpreters was the codification of design moves as grammar rules. Those that went from formalism to implementation, and especially to those used by other than their authors [24 15, 10, 11, 1, 12] made this codification active and thus, at some level, dealt with design space. In every case except for SEEDCONFIG [12] and SEED-LOOS [12], the strategy was minimal: the design space was represented either through (a subset of) the language of designs generated by a grammar or primarily through strands of derivation taken one at a time.

Codification is, at least in part, a successful amplification strategy. But just how does it work for the designer? What assurances and efficacies does it provide?

Authors such as Flemming [15], Archea [16], and Bruton [25] describe a mode of work with rule systems (used formally with an interpreter or informally as a metaphorical guide to action) in which the designer acts as author to the rule system. The account goes that the designers work in circles by making rules, using them, observing their actions, modifying the rules and reentering the circle by using them again. Bruton [25] captures this process especially well in the title of his thesis: A Contingent Sense of Grammar. The measure of rule utility is what it generates now and perhaps in future contexts. In general, we cannot assure formal properties 
in the utterances of a grammar. We might like to think that generative design holds forth the promise of operating within a distinct space of possibilities, for example, the space of well-formed Gaudian surfaces, but cannot guarantee this space. The space is implicit in the given rules, which induce appropriate designs or designs that can easily be ruled out. But where do rules arise? When we presume that a generative system is restricted to a well-formed subset of the possible Gaudian surfaces, we do so on the basis of past moves in a freer design space. We have, in general, no device for ensuring either that we capture all well-formed Gaudian surfaces or that we do not generate ill-formed surfaces along with the good ones. All we can do is temper our expectations with experience in the more open context in which we, or others, built the rules. In this more fluid context, ultimately the unrestricted design space, a trajectory records the co-option of design features and their assignments to roles. Rules ossify such experience. We have confidence in them to the extent that we can reinterpret the resulting designs ourselves, can follow the logic of the design space trajectory or have enumerated enough outcomes to feel confident in future explorations. Each of these sources of surety is an illusion. Re-interpretable designs arise in weak representations that defer explicit commitments to the viewer and thus surrender potential ground for computational assistance. Understanding design space trajectories privileges the easily explainable over the creatively explored. Confidence in numbers is a simple recapitulation of the general logical error of induction of general laws from specific facts.

The accounts generated in exploration are thus necessarily both constructive and contingent. It is not clear that you can provide a computationally tractable and humanly understandable mechanism that mimics set theoretical selection, that is, selection based on the interpretations of designs. In a constructive world, be it evolution or design, accessibility is the only sound concept for discovery. Accessibility derives not only from the operations at hand in the design representation, but also from the reuse of recurring patterns in the threads of actuality that trace out the explicit design space - the space of visited designs. Reuse of threads is the intuition behind, for instance, case based reasoning. However, from a design space explorer perspective, the case lies not in the individual designs but rather in the design space trajectories embedded in an explicit design space.

\section{Representation of Design Space}

A core feature of system designs for design space exploration is explicit representation of the space of discovered designs (the explicit design space). It necessarily inherits the properties of the task environment - designer action by search in a problem space - that it must serve. As we argued above, these include intentionality, partiality, Vastness, and the attendant primacy of accessibility over possibility. Further, a design space representation would seek to support one or more of the strategies outlined in Section 3 above.

What are the implications for design space representation? All such must arise against the constraints that a useful representation must be simultaneously understandable to its users and computable by a machine. Understandability may take many forms, but a chief strategy is to present to the user in terms of the tasks being undertaken, in the case of design space exploration, this is in terms of all the above-mentioned elements of its task environment and some, or all, of the amplification strategies. Computability means that we must carefully attend to using only algorithms and representations that work within the finite resources available to computation.

We call out some principal constraints from both understandability and computation. Work by search in a problem space immediately implies that some of the possible states will have been visited and some not. The visited ones are accessible by recall and subject to interstate comparisons. The unvisited states are possible and may become accessible through future acts of exploration. We distinguish these two classes of states by the designation of two subsets of design space. The implicit space is that made possible by the generative mechanism. The explicit space comprises those states that have been visited, in the current or an available past exploration episode.

The Vastness of design space is a permanent constraint. Examples of Vast spaces are the game tree of chess or the collection of all possible five-hundred-page ASCII manuscripts. The latter has more states that available protons in the universe. The fact is more often appreciated than the converse: that these spaces are in fact finite, are composed of finite objects and are reachable by finite computations. The upshot of Vast spaces, composed of finite elements, is that there are clear shortest path trajectories in the design space, and that such trajectories are generally tractable. For example, a book in the library of Babel [26] is reachable in a year to anyone prepared to type a couple of pages a day. However, to consider the contents of the same 
library is to boggle the mind. There is no class of English literature, or literature in any language, for example, biographies of the reader, that it does not house in Vast quantities. Therefore, we may be reasonably extravagant with algorithms that process trajectories in the design space, since they deal with tractable numbers of elements, while we must strenuously avoid algorithms that attempt brute force work against anything but a Vanishingly small fraction of the design space. Design space exploration thus inevitably mixes human intervention with machine generation; it is a mixed-initiative enterprise.

The term mixed initiative has a specific meaning in the wider literature, which we adopt without change here. It refers to the understanding of interaction with a system in terms of the communication that would occur were the system a colleague. Interaction changes from issuance of commands and reception of results to a multi-level system of dialogue, in which agents communicate within a domain to coordinate tasks and achieve goals [27, 28]. An implication for design space explorers is that the opportunities for external access to any generative mechanism need to be fine-grained. This is not a claim that all interaction must be fine-grained, just that it can be. In a Vast space task control becomes crucial - search is intractable, but movement along specific paths is efficient. Conversely, given a mechanism that can actually do much of the hard work of developing designs along given paths, the mechanism needs, at times, to retain initiative.

In the mixed-initiative enterprise of design space exploration, there are at least two important sources of clarity to exploit. The first is navigation, the second recombination.

Navigation is cognate movement through a space. It involves both moving along and understanding your trajectory. Navigation in implicit and explicit spaces is subject to the same constraint: it can only be done in reference to what is already known. This constraint manifests differently in each space. In implicit space, a navigator stands at the frontier of the explicit and must base action on some location on that frontier. She has in hand a set of operators, and some (necessarily fallacious, but heuristically useful) knowledge about what those operators might do. In explicit space, a designer has a richer context of reference. She can move from known state to known state along explicit paths that express some measure of relatedness. She can search through indexes of design state properties.

A flaw in standard rule-based accounts of design space exploration in implicit space is that the usual formulation of rules cast navigation solely in terms of derivation; thus putting the landscape of the explicit space forever beyond the sight of the navigator. Applying rules of the form $\alpha \rightarrow \beta$ involve removing a transformed version of $\alpha$ from the design and substituting for it an identically transformed version of $\beta$. This means that the granule of movement specified in a rule can go from one point in a design space to another arbitrary point irrespective of any underlying design space order. Rules of the form match and apply create a similar screen between derivation and design space structure to the extent that the apply part of the rules does not create an explicit account of its movement through the structure. Recasting the devices of navigation from rules to operations that make explicit reference to underlying structure permits us navigators to know more about the paths we have taken [4].

Recombination generalises the notion of Copy-andApply-Tendril argued above as a natural analogue to Copy-and-Paste in more conventional systems. Recombination permits the application of past navigation to future explorations. For example, we may splice together trajectories, or reorder the steps along a trajectory. A given design space representation will make a variety of such operations feasible, but we require certain regularities in the representation of design states that allow us to cheaply and robustly reapply design transformations, and to recognise some monotonically increasing properties along the trajectories.

Against the Vastness of the design space, it is clear that we cannot hope to exhaustively optimise. Even a Vanishingly small subclass of the design space is, in general, also Vast, so that design problems are examples of what Simon [20] calls satisficing. For example, you can only expect to arrange a Vanishingly small fraction of the competent autobiographies contained in the library of Babel, and those that you will reach will be by refinement of your original attempt. Therefore, it is crucial that the organization of the explored space makes possible the reuse of drafts.

If as we argued above, the best accounts of intent will necessarily include the decision history of design process, then narrative becomes an important part of a design system. The bet here is that telling stories of design decisions, including the decisions foregone, begets new understanding. Efficient navigation and recombination reinvigorate such narrative. To explain the telos of a design, simply replay an account of its creation. If the navigation mechanism embodies structure other than derivation, for example, plausible explanations other than those followed by the designer might be available and such explanations could just be interesting. Navigation and recombination reify storytelling as a design tool. 
Implicit space is necessarily a Vanishingly small part of design space, but that does not mean that it is small in computational terms. Over design episodes on a project and multiple projects it is likely to grow to very large size. This puts a premium on methods of recall that are both efficient in computational resources and effective to the designer. Similarity is the usual term used for such recall, and is the stock-in-trade of the large case-based reasoning literature. Again we call on a necessity for regularities in design space to both formalize and make efficient notions of similarity-based recall. An insight is to use the structure of the design space itself as the recall mechanism. To recall a past design, put an approximation of part of it into a design space. The designs proximal to it will be similar by the measures implied by design space structure.

The calls for navigation richer than derivation, robust recombination, recapitulation through replay and content-based recall all imply a primary space structuring mechanism other than rewrite rules (though rewrite rules could be built on top of such a mechanism). We are aware of at least one such computationally efficient mechanism, and this is based on a formalism known as typed feature structures. The essential information structuring relation here is subsumption, that is, a relation of information specificity. One state subsumes another if it contains strictly less information that the other. With some restrictions on the kinds of information carried (notably functional values for features), subsumption can be computationally efficient. Further, the formalism provides a means of codification and generation involving type hierarchies as an analogue to rewrite rules. It turns out the consequent style of expressing design information is familiar in computational design circles. It is essentially that of constraint languages in which users build descriptions of collections of objects as networks in which the arcs express either constraints or equality amongst variables depending on the view taken. In constraint languages, the utterances are used directly as design descriptions. In typed feature structures, they effectively become the rules that control generation through a resolution process, specifically called $\pi$-resolution.

Woodbury et al. [5] introduced the typed feature structures representation to design space exploration, argued its relevant properties, and showed how it could be used to model simple housing designs.

Burrow and Woodbury [29] gave precise definition to the typed feature structures representation as adapted from Carpenter [6], and showed how to make the $\pi$ resolution incremental. An incremental algorithm in which each stage of the resolution process is open to user interaction is a crucial property for a mixedinitiative design space explorer.

Woodbury et al. [4] introduced a set of fundamental operators over typed feature structure subsumption networks and showed how these could be used to generate explicit design spaces and navigate design spaces in general.

In his $\mathrm{PhD}$ thesis, Chang [7] showed how the typed feature structures representation could be extended to include objects, such as solids, that have indefinite subparts. He showed simple examples of generation using the extended representation.

Datta's thesis [28] constructs a mixed-initiative model for interacting with subsumption-based representations in general and the extended typed feature structures representation in particular.

In his upcoming thesis, Burrow [30] has shown that efficient design spaces can be organized around typed feature structures and $\pi$-resolution. For the first time, a formalism simultaneously captures generation, navigation, recombination, recapitulation and recall.

The typed feature structure representation provides a computationally efficient substrate for design space exploration. To do this it imposes rigorous constraints on the properties of a representation - it introduces significant properties that are exogenous to the task of simply representing a design. The representation supports the properties we argued for in the discussion of the design space exploration domain in Section 2, namely abstraction above the program, partiality, intentionality, and the ability to provide both strong and weak representations. Its form of codification is more declarative than conventional rules - a user describes structure rather than operations. Its algorithms are incremental at a fine grained level of the generation process, thus allowing for mixed-initiative interaction at almost any stage of the generation and navigation process.

The representation is young. In front of it lies the sorts of effective, medium scale demonstrations achieved by Heisserman [11], Woodbury et al. [31], Carlson [32], Carlson and Woodbury [33], Akin et al. [12] and Harada [34]. Far in front of it lies serious industrial application [1]. It is though, the first representation that gives system designers a meaningful handle on the primary object of research in the field, the design space.

Without knowing the periodicity and lifetime of patterns in the design space, it is better to equip the designer with an explorer that can be reshaped and tendrils that can be reused as patterns emerge in the work of constructing threads of actuality. This is another reason why design space explorers are mixed initiative 
systems - not viewers of ready-made designs for a human. They are systems requiring reflective thought from designers.

\section{Summary}

To take the metaphor of design space exploration seriously is to admit the design space as the primary object of research. Possible structures for design space are conditioned by models of exploration behaviour by designers, by choices of strategies for amplifying designer action and by the limits imposed by both computation itself and our knowledge of it. Formalisms for design space exploration must simultaneously accord with designer action, implement a useful amplification strategy and be both formalizable and computationally tractable.

\section{Acknowledgements}

Prior publication of parts of this article appeared in [35] and [36].

This work was partially supported through the Australian Research Council Large Grants Scheme, the Australian Research Council Small Grants Scheme, the National Science and Engineering Research Council Research Grants Program, the Advanced Systems Institute of British Columbia Provincial Research Fellowships Program and the University of Adelaide.

\section{References}

[1] Heisserman, J., Callahan, S., and Mattikalli, R., A design representation to support automated design generation. In Gero, J., editor, Artificial Intelligence in Design 00. Key Centre for Design Computing, Kluwer, 2000.

[2] Chien, S-F and Flemming, U., Information navigation in generative design systems, In Yu-Tung Liu, J-Y Tsou, J-H, editor, CAADRIA 97, volume 2, pages 355-366, Computer Aided Architectural Design Research in Asia, National Chia Tung University, Taiwan, 1997.

[3] Chien, S-F., Supporting Information Navigation in Generative Design Systems, $\mathrm{PhD}$ thesis, Department of Architecture, Carnegie Mellon University, 1998.

[4] Woodbury, R., Datta, S., and Burrow, A., Erasure in design space exploration. In Artificial Intelligence in Design 2000, pages 521-544, Worcester, Massachusetts. Key Centre For Design Computing, 2000.

[5] Woodbury, R. F., Burrow, A. L., Datta, S., and Chang, T.W., Typed feature structures in design space exploration. Artificial Intelligence in Engineering Design and Manufacturing, 13(4), pp. 287-302, 2000.

[6] Carpenter, B., The Logic of Typed Feature Structures with applications to unification grammars, logic programs and constraint resolution. Cambridge Tracts in Theoretical Computer Science. Cambridge University Press, 1992.

[7] Chang T.W., Geometric Typed Feature Structures: Toward Design Space Exploration, PhD Thesis, The University of Adelaide, 1999.
[8] McDermott, J., R1: A rule-based configurer of computer systems. Artificial Intelligence, 19(1):39-88, 1982.

[9] Forgy, C., OPS5 User's Manual. Department of Computer Science, Carnegie-Mellon University, Pittsburgh, Pa. 15213, 1981.

[10] Flemming, U., Knowledge representation and acquisition in the loos system. Building and Environment, 25(3):209-219, 1990.

[11] Heisserman, J., Generative geometric design. IEEE Computer Graphics and Applications, 14(2):37-45, 1994.

[12] Akin, O., Aygen, Z., Chang, T.-W., Chien, S.-F., Choi, B., Donia, M., Fenves, S. J., Flemming, U., Garrett, J. H., Gomez, N., Kiliccote, H., Rivard, H., Sen, R., Snyder, J., Tsai, W.-J., Woodbury, R., and Zhang, Y., SEED: A Software Environment to support the Early phases of building Design. The International Journal of Design Computing. 1997. Accessed 15 June 2004 at

http://www.arch.usyd.edu.au/kcdc/journal/index.html.

[13] Dennett, D. C., Darwin's Dangerous Idea: Evolution and the Meanings of Life. Simon \& Schuster, 1995.

[14] Gould, S.J., Wonderful Life: The Burgess Shale and the Nature of History, W.W. Norton, 1990.

[15] Flemming, U., The role of shape grammars in the analysis and creation of designs. In Kalay, Y., editor, Computability of Design, Principles of Computer-Aided Design, chapter 12, pages 245-272. Wiley Interscience, New York, N.Y, 1987.

[16] Schon, D., The Reflective Practitioner: How Professionals Think in Action. Basic Books, New York, 1983.

[17] Archea, J., Puzzle-making: What architects do when no one is looking. In Kalay, Y., editor, Computability of Design, Principles of Computer-Aided Design, chapter 2, pages 37-52. John-Wiley and Sons, New York, N.Y, 1987.

[18] Wright, F. L., An Autobiography. Hyperion Press, 1945.

[19] Woodbury, R.F., Grammatical Hermeneutics, Architectural Science Review, 36(2), pp. 53-64, 1993.

[20] Simon, H. A., The Sciences of the Artificial - Second Edition. MIT Press, Cambridge, MA, 1980.

[21] Newell A. and Simon H.A., Human Problem Solving, Prentice-Hall Inc., Englewood Cliffs, N.J., 1972.

[22] Akin, O., The Psychology of Architectural Design. Pion, London, 1986.

[23] Knight, T., Languages of designs: from known to new. Environment and Planning B: Planning and Design, 8:213238, 1981.

[24] Flemming, U., Wall representations of rectangular dissections and their use in automated space allocation. Environment and Planning B: Planning and Design, 5:215232, 1978.

[25] Bruton, D., A Contingent Sense of Grammar, PhD Thesis, The University of Adelaide, 1997.

[26] Borges, J., Labyrinths: Selected Stories and Other Writings, chapter The Library of Babel. New Directions, 1962.

[27] Allen, J., Mixed Initiative Interaction, Proc. IEEE Intelligent Systems, 12(6), 14-23, 1999.

[28] Datta, S., Unfolding Design Spaces Interactively, PhD Thesis, The University of Adelaide, 2004.

[29] Andrew Burrow and Robert Woodbury. $\pi$-resolution in design space exploration. In Godfried Augenbroe and Charles Eastman, editors, Computers in Building: Proceedings of the CAADFutures'99 Conference, pp 291-308, Kluwer Academic Publishers, Atlanta, Georgia, June 7-8, 1999. 
[30] Burrow, A., Typed Feature Structures and Design Space Exploration. PhD thesis, The University of Adelaide. to appear, 2004.

[31] Woodbury, R. F., Radford, A. D., Taplin, P. N., and Coppins, S. A. Tartan worlds: A generative symbol grammar system. In D. Noble and K. Kensek, editors, ACADIA 92, pages 211-220, Charleston, SC, October 1992.

[32] Carlson, C. Grammatical Programming: An Algebraic Approach to the Description of Design Spaces. PhD Thesis, Carnegie Mellon University, 1993.

[33] Carlson, C. and Woodbury, R. Hands-on exploration of recursive patterns. Languages of Design, 2:121-142, 1994.

[34] Harada, M., Witkin, A.P., Baraff, D., Interactive physically-based manipulation of discrete/continuous models. SIGGRAPH 1995 : 199-208, 1995.

[35] Woodbury, R. and Burrow, A., Design spaces: The forgotten artifact. In Burry, M., Datta, S., Dawson, A., and Rollo, J., editors, Proceedings of the Third International Conference on Mathematics and Design 2001, pages 56-62, Geelong, Victoria, Australia, 2001.

[36] Robert F. Woodbury and Andrew Burrow, Notes on the Structure of Design Space, International Journal of Architectural Computing, 1(4): 517-532, 2004. 